\title{
IMPACT OF CALIBRATION CURVE PRECISION ON RESULTS OF IMRT VERIFICATION WITH EBT3 FILMS
}

\author{
Tereza Hanušová ${ }^{*}$, Simona Buryšková ${ }^{1,2}$ \\ ${ }^{1}$ Faculty of Nuclear Sciences and Physical Engineering, Czech Technical University in Prague, Prague, Czech Republic \\ ${ }^{2}$ Mathias Lerch Gymnasium, Brno, Czech Republic
}

\begin{abstract}
This study aims to establish proper methodology for IMRT treatment plan verification using EBT3 film dosimetry. This preliminary part focuses on proper calibration curve fitting and its impact on the results of gamma analysis. First, scanner and film properties were tested in order to estimate their important characteristics. The films in use were Gafchromic EBT3 together with EPSON Perfection V7Oo Photo scanner. Three sets of calibration points were obtained for the EBT3 film. Irradiation was carried out on a Siemens Artiste linear accelerator in the Thomayer Hospital in Prague, using the photon energy 18MV. Film pieces of approximately $6 \times 6 \mathrm{~cm}^{2}$ were irradiated with 16 doses ranging from o to 12 Gy in an RW3 slab phantom in reference conditions. Three sets of films of the same calibration doses were irradiated in three subsequent weeks and scanned in two different ways, changing the scanned region of interest. The mean value of each scanned piece of film was taken as one calibration point. Different calibration curves were obtained in MATLAB, Excel and FilmQA Pro. Both polynomial fits with a fourth degree polynomial and rational functions as proposed in FilmQA Pro were assessed. Different approaches to curve fitting were examined: use of all measured points; omission of bad points from the calibration curve; average values of all sets of calibration points. The single channel approach was used in all cases, using the red channel. Other films were irradiated with an IMRT plan for prostate and lymph nodes. The plan contained 5 fields with $18 M V$ photon beams and each field was tested separately with the gantry position at $O^{\circ}$. Films were placed into the coronal isocentric plane of an $R W_{3}$ slab phantom. They were scanned using the same protocol as for the calibration films, fitted with the different calibration curves in MATLAB and evaluated in OmniPro I'mRT software using gamma analysis with $4 \% / 3 \mathrm{~mm}$ criteria. Different gamma pass rates for the very same fields could be seen when different calibration scenarios were applied. These differences in gamma pass rates were not negligible, even when the estimated precision of calibration curves was similar. Best results were obtained with a MATLAB curve when three sets of calibration films were averaged out. This reduces the impact of film and scanner inhomogeneity and other deviations that can occur during film handling. Careful investigation into the calibration curve assessment should be carried out when implementing film dosimetry for IMRT or VMAT verification into the clinic to achieve the best results. Otherwise, the results of plan verification can be dependent on the calibration curve and can fail tolerance limits, even though the predicted and measured dose distributions agree reasonably well if measured properly.
\end{abstract}

Key words: Radiochromic film dosimetry, film calibration, IMRT verification, gamma analysis

DOI: $10.21175 /$ RadProc.2016.13

\section{INTRODUCTION}

Verification of Intensity Modulated Radiation Therapy (IMRT) and Volumetric Modulated Arc Therapy (VMAT) plans is performed either by point dose measurements with a cylindrical ion chamber or by dose distribution measurements with electronic detectors (ion chamber or diode arrays), or alternatively with film. Usually, both approaches are combined in clinical practice and in many clinics this process is performed for each new IMRT or VMAT plan. Electronic detectors usually have limitations in spatial resolution and verification results can sometimes fail to meet tolerance limits just due to this fact. Film dosimetry with Gafchromic EBT3 films seems like a promising alternative but the process of film handling and calibration is very demanding and time consuming, so it has not yet replaced electronic detectors for IMRT and VMAT verification. Many works have been published (for example [1]-[5]) on the topic of proper film handling and calibration but each site must take into account their own working conditions and equipment and should adjust guidelines published in literature. This preliminary study aims to establish proper methodology for IMRT treatment plan verification using EBT3 film, which will then be used for further investigations into pseudo $3 \mathrm{D}$ dosimetry. Interesting findings about the impact of calibration curve precision on the results of gamma analysis performed on films irradiated with clinical fields are published in this article.

\section{SCANNING PROCESS}

\subsection{Scanning parameters}

EPSON Perfection V700 Photo scanner was used in this study together with EBT3 films from the batch number 09151403. All calibration and clinical films were taken from the same box, so results are not influenced by possible differences.

Scanning parameters were as follows: Professional mode, Color negative film, Film with film area guide,

* tereza.hanusova@fjfi.cvut.cz 
48-bit colors, 72 dpi, uncorrected images saved in tiff format, portrait orientation. Films were placed exactly in the center of the scanner area with the help of markers and scanned in transmission mode. The same orientation of all calibration and IMRT films was kept at the accelerator and at scanning. All films, calibration and clinical, were scanned 72 hours after irradiation. The red channel data were used in all cases. Proper scanner warm-up was ensured by scanning 5 blank scans prior to film scanning.

Among the important characteristics, scanner and film homogeneity and reproducibility of the scanning process should be assessed.

\subsection{Scanner and film homogeneity}

Homogeneity was determined for the scanner itself by scanning a small piece of unirradiated film (approximately $4 \times 4 \mathrm{~cm}^{2}$, excluding the film edges from the scanning region of interest - ROI) in various places over the scanner area. The mean value of scanner response over this $4 \times 4 \mathrm{~cm}^{2}$ area for each scanned image was denoted $M$. Homogeneity over a given region was calculated as

$$
H_{s}=\frac{M_{\text {max }}-M_{\text {min }}}{M_{\text {mean }}}
$$

It should be pointed out that $M$ would be constant for the whole scanner area if the scanner was ideally homogeneous, and $H_{s}$ would be zero in this case. The central region of the scanner $\left(16 \times 16 \mathrm{~cm}^{2}\right)$ was found to be homogenous with a homogeneity index of $2.53 \%$. When the whole scanner area was assessed, the homogeneity index increased to a value of $7.04 \%$. However, the central area is always used for scanning.

Then film and scanner homogeneity was combined by scanning a larger unirradiated piece of film (ROI of $6 \times 6 \mathrm{~cm}^{2}$ ) and applying a median smoothing filter to the scanned image with a matrix size of $5 \times 5$ pixels (which was then done for film evaluation as well otherwise, inhomogeneity would be even larger). The scanned zero dose image before smoothing is shown in Figure 1. Each pixel value of the smoothed matrix was denoted $M$ and homogeneity over this ROI was calculated as

$$
H_{s f}=\frac{M_{\text {max }}-M_{\text {min }}}{M_{\text {mean }}}
$$

Homogeneity index in this case was found to be worse, namely $18.26 \%$.

Scanner and film homogeneity combined with linear accelerator (linac) field homogeneity (Siemens Artiste $18 \mathrm{MV}$ beam, $10 \times 10 \mathrm{~cm}^{2}$ field size) was assessed by scanning calibration films irradiated with different doses ranging from 0.3 to $12 \mathrm{~Gy}$. After the same process as in the previous step, the combined homogeneity was between 6.73 and $16.71 \%$ with better results obtained with higher doses.

The combined homogeneity was found to be acceptable if doses of at least 1 Gy were used for film irradiation. It is clear that zero dose and very low doses should be avoided with radiochromic film dosimetry. It should also be noted that film inhomogeneity could influence the results of gamma analysis in a way that is described in [6]. For this type of study, however, it is unimportant.

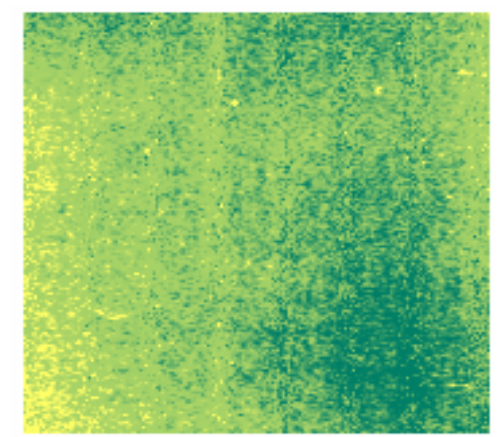

Figure 1. Film and scanner inhomogeneity summed together in a zero dose film image; image scaled between the minimum and the maximum scanner value

\subsection{Scanner reproducibility}

Short term reproducibility of the scanning process was assessed by scanning the same piece of film (doses of $0.3,1,2,5$ and $8 \mathrm{~Gy}$ ) five times in sequence and determined as the relative error of the mean value. The maximum value of short term reproducibility found for the above mentioned doses was $0.24 \%$. Long term reproducibility was assessed by scanning the same piece of film (doses of $0.3,1,2,5$ and 8 Gy) in several subsequent days 1 month after irradiation, when the signal of the film itself was believed to be stable. Long term stability of the scanner was found to be less than $0.23 \%$ for all doses between 0.5 and 8 Gy. However, for $0.3 \mathrm{~Gy}$ it was as bad as $20 \%$. Again, very low doses should be avoided with radiochromic film dosimetry.

Having understood the performance of the films and scanner, it was decided to use doses of $2-3$ Gy (thus, maximum dose in a field usually around $4.5 \mathrm{~Gy}$ ) to irradiate films with clinical IMRT fields. Higher doses would be impractical due to very long irradiation times. Lower doses can also be excluded from evaluation using a dose threshold for gamma analysis, the level of which can be derived from the film and scanner properties analysis.

\section{FILM IRRADIATION AND SCANNING}

\subsection{Irradiating and scanning calibration films}

Irradiation of calibration films was carried out on a Siemens Artiste linear accelerator in the Thomayer Hospital in Prague, using the photon energy 18MV. Film pieces of $6 \times 6 \mathrm{~cm}$ were irradiated with 16 doses ranging from o to $12 \mathrm{~Gy}(\mathrm{O}, 0.3,0.5,1,1.5,2,2.5,3,3.5$, $4,5,6,7,8,10$ and $12 \mathrm{~Gy}$ ) in an RW3 slab phantom in reference conditions (10 x $10 \mathrm{~cm}^{2}$ filed, $10 \mathrm{~cm}$ depth).

Three sets of calibration films were obtained: three series of films of the same calibration doses were irradiated in three subsequent weeks. Linac output measured with an ion chamber over that time period changed only within $0.5 \%$ and this variation was not taken into account. Moreover, set no. 1 was scanned in two different ways, changing the scanned region of interest from $5 \times 5 \mathrm{~cm}^{2}$ to $5.8 \times 5.8 \mathrm{~cm}^{2}$ to see if this can also have an impact on the calibration curve and evaluation of films. In all cases, film edges possibly damaged by cutting were excluded from the scanned region of interest. The different calibration sets of films are described in Table 1 for summary. The mean or the 
median value of each scanned image was taken as one calibration point.

Table 1. List of calibration film sets

\begin{tabular}{|l|l|}
\hline Set no. & Film set description \\
\hline 1 & $\begin{array}{l}\text { Measured in Week } 1, \text { central area } \\
\text { of film scanned }(5 \times 5 \mathrm{~cm})\end{array}$ \\
\hline 2 & $\begin{array}{l}\text { Measured in Week 1, larger area } \\
\text { of film scanned }(5.8 \times 5.8 \mathrm{~cm})\end{array}$ \\
\hline 3 & $\begin{array}{l}\text { Measured in Week 2, larger area } \\
\text { of film scanned }(5.8 \times 5.8 \mathrm{~cm})\end{array}$ \\
\hline 4 & $\begin{array}{l}\text { Measured in Week } 3, \text { larger area } \\
\text { of film scanned }(5.8 \times 5.8 \mathrm{~cm})\end{array}$ \\
\hline
\end{tabular}

\subsection{Irradiating and scanning films with clinical} fields

Films were irradiated with an IMRT clinical step and shoot plan for prostate and lymph nodes prepared for a Siemens Artiste photon energy 18 MV. Treatment planning was performed in $\mathrm{XiO}$ version 4.80 . The plan contained 5 fields with $18 \mathrm{MV}$ photon beams, fields were distributed evenly in the whole $360^{\circ}$. General High Smoothing was applied during optimization and other optimization parameters were left default. The prescribed dose was 44 Gy in 22 fractions. Each field was tested separately with the gantry position at $0^{\circ}$.

Films were placed into the isocentric coronal plane into an RW3 slab phantom at the depth of $10 \mathrm{~cm}$ (the same setup as for the calibration films). The size of film sheets used for testing the clinical fields was 8.4 x 6.8 $\mathrm{cm}^{2}$ in order to measure the important part of each field but at the same time avoid too low doses that proved not to be measurable with sufficient precision. Each piece of film (each field of the plan) was irradiated 10 times in order to reach doses around $2-$ 3 Gy on film. These films were scanned using the same protocol as for the calibration films. The only difference was the scanning region of interest, which was larger than the films themselves, so as to keep markers on the film visible in the image for adjustment of film rotation in the evaluation software for gamma analysis.

\section{Calibration and EVAluation PROCESS}

\subsection{Obtaining different calibration curves}

Different calibration curves were obtained in MATLAB, Excel and FilmQA Pro. Both polynomial fits with a fourth degree polynomial and rational functions as proposed in FilmQA Pro were assessed. In MATLAB, the polyfit function was used to fit a $4^{\text {th }}$ degree polynomial to the measured calibration points. This function uses the Vandermonde matrix and solves it via $\mathrm{QR}$ factorization to calculate the polynomial coefficients. Centering and scaling was used to improve numerical properties of the system. In Excel, the $4^{\text {th }}$ degree polynomial was obtained with the help of the LINEST function. It uses the least squares approach solving a set of linear equations. Then polynomial coefficients obtained with LINEST were processed by the Excel Solver tool, but it did not further improve the results. Film images were processed in MATLAB to obtain calibration points for MATLAB and Excel.
Both mean and median values of the scanned calibration images were taken as calibration points to see if film inhomogeneity would play a more important role in one of these approaches. All measured calibration points were used for some curves. In other curves, some points were excluded (points that deviated the most from the intended calibration curve fit). Also, different calibration sets were averaged out to obtain one average curve. Even different precision of curve coefficients (different number of decimal places) was assessed when fitting the measured IMRT fields with the calibration curve.

As for FilmQA Pro, it offers different types of calibration functions: reciprocal linear, rational linear, rational quadratic, rational cubic and exponential. These should, according to software manufacturers, better suit the real behaviour of films with increasing dose. However, they gave very poor results in terms of gamma analysis (see Results and discussion) and are not further mentioned here. Nonetheless, calibration points obtained as mean values of film images processed in FilmQA Pro in the red channel were fitted with $\mathrm{a} 4^{\text {th }}$ degree polynomial in Excel and are mentioned as curve no. 6 in Table 2.

Table 2. List of investigated calibration curves ( $4^{\text {th }}$ degree polynomials); the best result marked in grey

\begin{tabular}{|l|l|}
\hline $\begin{array}{l}\text { Curve } \\
\text { no. }\end{array}$ & Type of curve \\
\hline 1 & $\begin{array}{l}\text { Excel, median scanner value, average of } 3 \\
\text { calibration film sets }\end{array}$ \\
\hline 2 & $\begin{array}{l}\text { Excel, mean scanner value, average of } 3 \\
\text { calibration film sets }\end{array}$ \\
\hline 3 & $\begin{array}{l}\text { Excel, mean scanner value, average of } 3 \\
\text { calibration film sets, less points used for fit }\end{array}$ \\
\hline 4 & $\begin{array}{l}\text { Excel, median scanner value, average of } 3 \\
\text { calibration film sets, less decimal points in } \\
\text { curve coefficients than Curve 1 }\end{array}$ \\
\hline 5 & $\begin{array}{l}\text { Excel, median scanner value, average of } 3 \\
\text { calibration film sets, less points used for fit }\end{array}$ \\
\hline 6 & $\begin{array}{l}\text { Excel, mean scanner values from FilmQA } \\
\text { Pro, film set no. } 3\end{array}$ \\
\hline 7 & MATLAB, mean scanner value, film set no. 1 \\
\hline 8 & MATLAB, mean scanner value, film set no.3 \\
\hline 9 & $\begin{array}{l}\text { MATLAB, mean scanner value, average of } 3 \\
\text { calibration film sets }\end{array}$ \\
\hline
\end{tabular}

The tested combinations that are shown in this paper are listed in Table 2 and the curve found to give the best results is marked in grey. Other combinations are discussed in the Results and discussion section.

\subsection{Evaluating clinical films with gamma analysis}

All measured IMRT fields were fitted with the different calibration curves in MATLAB using the function polyval and evaluated in OmniPro I'mRT software using gamma analysis. The gamma criteria were $4 \%$ dose difference and $3 \mathrm{~mm}$ distance to agreement, search distance $4.5 \mathrm{~mm}$, avoiding signals with lower doses than $10 \%$ of the maximal predicted dose. Relative gamma was performed and both the predicted and the measured dose distributions were normalized to the average value of the chosen region of interest. ROI was chosen so that film edges and film 
markers were excluded from evaluation. Predicted and measured dose distributions were matched with the help of film markers and with the help of $\mathrm{X}$ and $\mathrm{Y}$ dose profiles. The dose grid of the predicted matrices was converted to the same pixel distance as for the scanned (measured) matrices (0.352778 $\mathrm{mm}$ ) using linear interpolation. Measured dose matrix was smoothed with a median filter of the size $5 \times 5$ pixels.

Predicted dose matrices used for evaluation were exported from TPS (treatment planning system) XiO version 4.80 . The clinical plan was recalculated into a virtual water phantom of the size $30 \times 30 \times 30 \mathrm{~cm}^{3}$ (both clinical plan measurements and calibration was done in an RW3 slab phantom, but supposing a virtual water phantom in the TPS, so that difference between water and RW3 material was comprised in the calibration curve). The XiO tool Modulation QA was used to export the predicted matrices for individual fields in ASCII format in the depth of $10 \mathrm{~cm}$ and SSD $90 \mathrm{~cm}$ and with gantry angle of $\mathrm{o}^{\circ}$ for all fields.

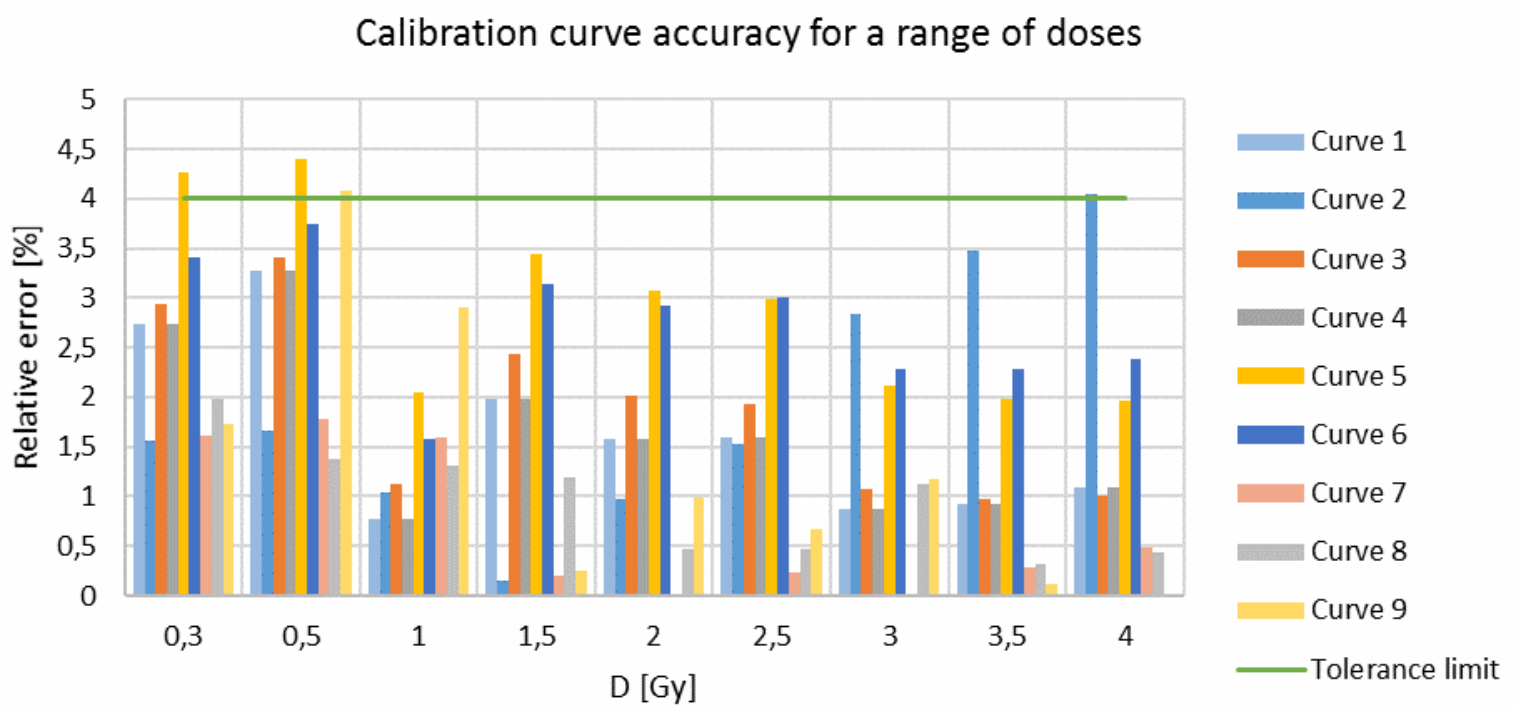

Figure 2. Accuracy of calibration curves when applied to films irradiated with known doses. The maximum tolerable discrepancy is $4 \%$ ( $4 \%$ dose difference criterion was used for gamma analysis)

Different calibration curves - gamma pass rates

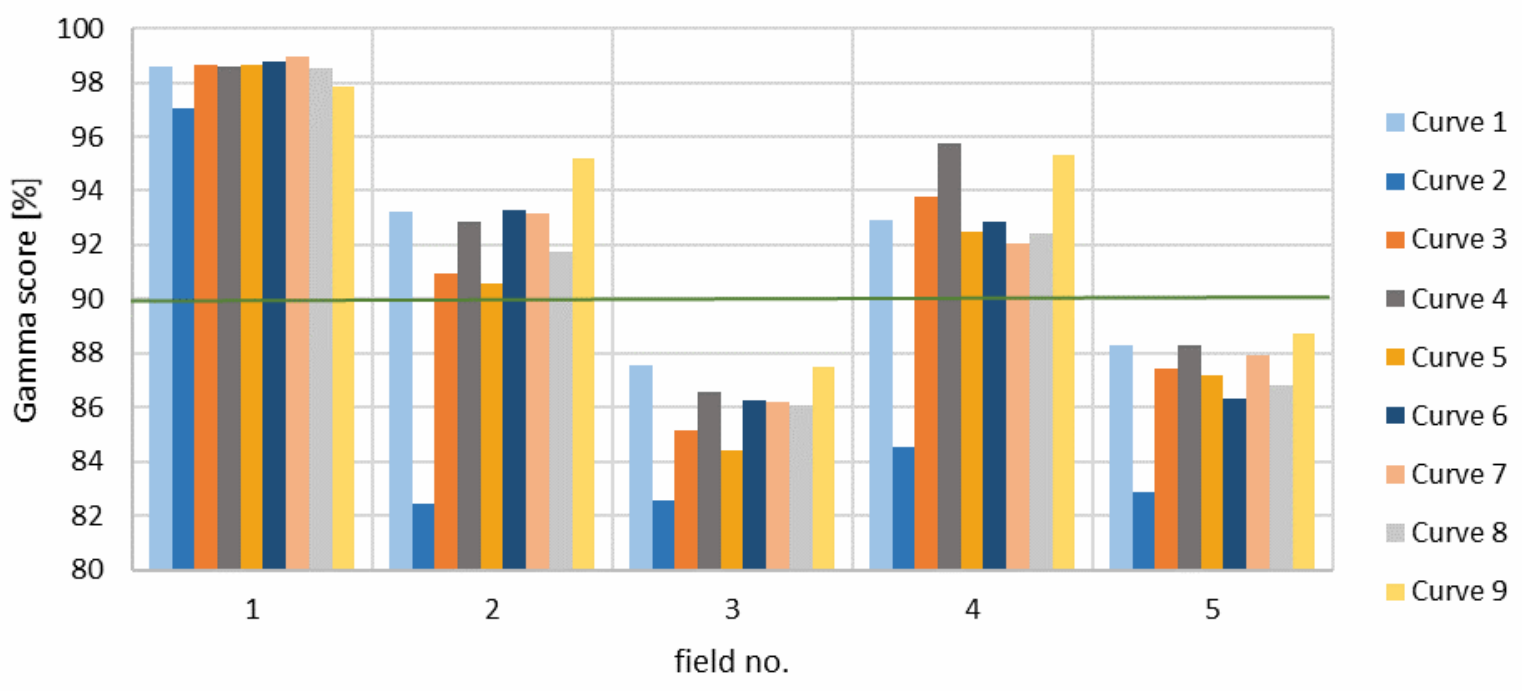

Figure 3. Gamma pass rates for the same 5 clinical fields obtained with 9 different calibration curves. Green line marks clinical tolerance limit. 


\section{RESUlTS AND DISCUSSION}

An example of a calibration curve obtained in MATLAB (namely curve no. 3) is shown in Figure 4. All curves (all calibration points) seem to overlap each other when judged visually, as can be seen in Figure 5 . Accuracy of the different calibration curves was evaluated with an independent set of films irradiated in the above described conditions with known doses. Linac output variation and field inhomogeneity was not taken into account because linac output was stable within $0.5 \%$ and homogeneity within $2.5 \%$ for the given energy and field sizes. Accuracy obtained for several different doses is plotted in Figure 2, showing the relative difference between the nominal dose and the one derived from the calibration curve for each calibration point. Most curves did not deviate more than $4 \%$ from the known dose for all dose points. Based on this, the dose difference criterion used in OmniPro Im'RT for gamma analysis was chosen to be $4 \%$ (even though a few curves exceed this value for low doses). It should be pointed out that the "known doses" were obtained with film again, which does not work well for low doses. The aim of Figure 2 is rather to illustrate the differences for the different calibration curves. Precision of curve fitting was also determined via the $\mathrm{R}^{2}$ coefficient of determination in Excel and via residual plots in MATLAB. There were only negligible variations among the different calibration curves.

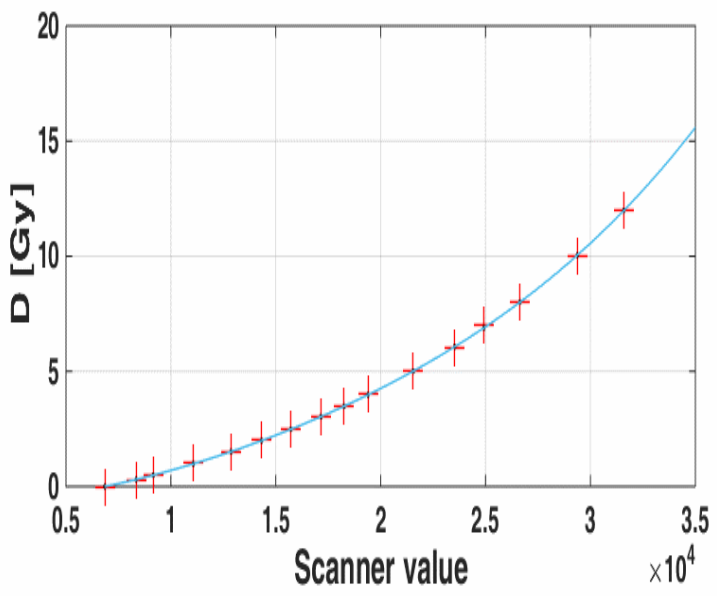

Figure 4. An example of a calibration curve obtained in MATLAB

Table 3 shows the average gamma pass rates for the clinical plan (averaged over 5 fields of the plan), obtained with the different calibration curves. Figure 3 shows gamma pass rates for the different calibration curves for all 5 fields individually. Curve 9, which is obtained in MATLAB as the average of calibration film sets no. 1, 2 and 3 (see Table 2), proves to give the best results. Not for all individual fields, as can be seen in Figure 3, but on average, as can be seen in Table 3 . (This plan was finally not used for patient irradiation due to unsatisfactory results of verification. These come from the complex anatomy of this particular patient. Fluence complexity is rather close to a complex head and neck clinical case).

Calibration film set no. 4 measured in Week 3 had to be excluded from evaluation due to very poor quality of the calibration curve. An unknown error occurred during film irradiation, scanning or handling that caused the results of this series of calibration films to be unreliable. Calibration curves obtained as rational functions in FilmQA Pro gave very poor gamma results when films irradiated with IMRT fields were fitted in MATLAB and evaluated in OmniPro I'mRT software. However, when IMRT fields were evaluated in FilmQA Pro software itself, gamma results were different and closer to the expected values. The cause of this effect has not yet been resolved due to lack of information on algorithms used in FilmQA Pro. Results are not shown here, as they are not comparable to results from OmniPro I'mRT software.

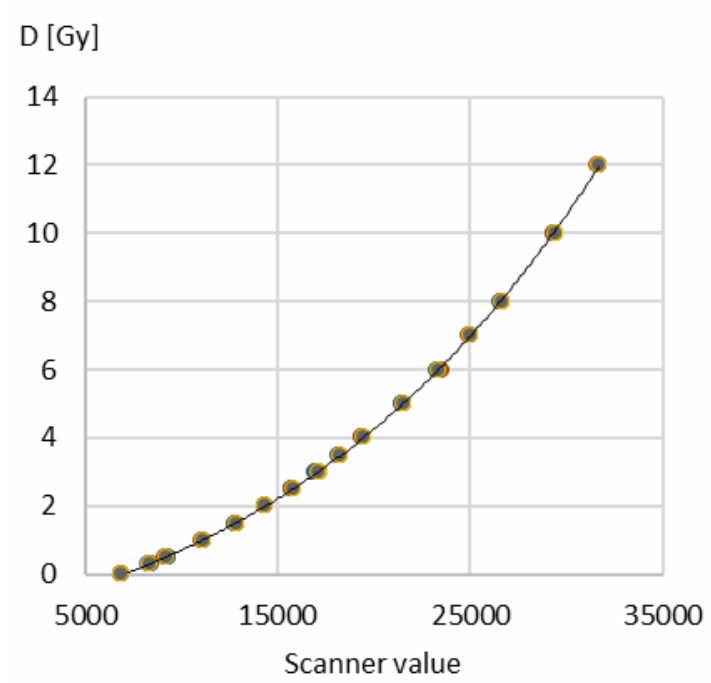

Figure 5. Nine different calibration curves - visually, all calibration points almost overlap and their fitted curves are very similar

Table 3 Average gamma pass rates for different calibration curves (averaged over 5 fields from the clinical plan); the best result marked in grey

\begin{tabular}{|l|c|}
\hline Curve no. & Gamma score [\%] \\
\hline 1 & 92.11 \\
2 & 85.88 \\
3 & 91.18 \\
4 & 92.41 \\
5 & 90.65 \\
6 & 91.50 \\
7 & 91.66 \\
8 & 91.12 \\
9 & 92.92 \\
\hline
\end{tabular}

The standard procedure for dose map verification in the Thomayer Hospital in Prague consists in measurements with the PTW seven29 detector array and evaluation in the PTW VeriSoft software. The tolerance limit is a gamma score of $90 \%$ - at least $90 \%$ of points in the dose distribution must meet the criteria of $3 \%$ dose difference and $3 \mathrm{~mm}$ distance to agreement. If the same tolerance limit is kept here, in Figure 3 it can be seen that in some cases this limit would be met and in some cases it would not, depending solely on the choice of calibration curve. Figure 3 aims to illustrate the differences between the calibration curves rather than to show if the tolerance 
limit was met. Better results are expected if the dose threshold for gamma analysis is increased (here it was only around $0.45 \mathrm{~Gy}$ - still too low with regard to the performance of films for low doses). Or if the doses used to irradiate films are increased. It is expected that results for field no. 3 and field no. 5 are influenced by lower doses present in these fields. Evaluation for all fields and calibration curves was done in exactly the same way, keeping all parameters the same. The same measured piece of film was fitted with different curves and these results were compared. So the differences could only be caused by variations in the calibration curves.

\section{CONCLUSIONS}

Different gamma pass rates for the very same pieces of film evaluated with different calibration curves could be seen. The accuracy of all investigated calibration curves was comparable, yet the differences in gamma pass rates were not negligible, even exceeding tolerance limits for some of the curves. Best results were obtained with a MATLAB curve when three sets of calibration films were averaged out. This reduces the impact of film and scanner inhomogeneity and other deviations that can occur during film handling.

Careful investigation into the calibration curve assessment should be carried out when implementing film dosimetry for IMRT or VMAT verification into the clinic to achieve the best results. Otherwise, results of plan verification can be dependent on calibration curve and can fail tolerance limits, even though the predicted and measured dose distributions agree reasonably well if measured properly.
Acknowledgement: This paper is a part of the research done within the students grant no. SGS15/217/OHK4/3T/14. Authors would like to thank their colleagues in the Thomayer Hospital in Prague for help with measurements, especially to Alžběta Lépesová. They would also like to thank Vladimír Dufek from the Czech National Radiation Protection Institute for valuable advice on film dosimetry.

\section{REFERENCES}

1. W. Crijns, F. Maes, U.A. van der Heide and F. Van den Heuvel, "Calibrating Page Sized Gafchromic EBT3 Films," Med. Phys., vol. 40, no. 1, pp. 012102-, Jan. 2013

2. V.C. Borca et al., "Dosimetric Characterization and Use of GAFCHROMIC EBT3 Film for IMRT Dose Verification," J. Appl. Clin. Med. Phys., vol. 14, pp. 158171,2013

3. P. Papaconstadopoulos, G. Hegyi, J. Seuntjens, and S. Devic, "A Protocol for EBT3 Radiochromic Film Dosimetry Using Reflection Scanning," Med. Phys., vol. 41, no. 12, pp. 122101-, Dec. 2014

4. A. Micke, D.F. Lewis and X.Yu, "Multichannel film dosimetry with nonuniformity correction," Med. Phys., vol. 38, pp. 2523-2534, May 2011

5. C. Fiandra et al., "Comparison of Gafchromic EBT2 and $\mathrm{EBT}_{3}$ for Patient-Specific Quality Assurance: Cranial Stereotactic Radiosurgery Using Volumetric Modulated Arc Therapy with Multiple Noncoplanar Arcs," Med. Phys., vol. 40, pp. 082105-, Aug. 2013

6. L.J. Schreiner, O. Holmes and G. Salomons, "Analysis and Evaluation of Planned and Delivered Dose Distributions: Practical Concerns with $\gamma^{-}$and $X^{-}$ Evaluations," J. Phys. Conf. Ser., vol. 444, no. 1, pp. o12016-, June 2013 